Submission ID: 43908

\title{
Study of Gas Hydrates in the Bering Sea Using Velocity and AVO-Analyses
}

\author{
V.V. Zhigulev* (Dalmorneftegeophysica JCS)
}

\section{SUMMARY}

The main seismic indication that points to presence of gas hydrates in the sedimentary section is a seismic reflector that roughly follows seafloor topography and crosscuts the reflections from lithologic boundaries and is known as "bottom simulation reflection" (BSR). To identify BSR in a seismic section where it is subparallel to lithologic boundaries, it is suggested to use velocity analysis that involves velocity spectrum computation and AVO analysis. It was determined that gas hydrate layer is recorded as a period of higher interval velocity values relatively to the overlying water layer and underlying sedimentary deposits. In the AVO results the BSR boundary shows itself as high wave dynamics with phase inversion (intercept attribute (I)), intensive positive anomaly (gradient attribute (G)) and negative anomaly (fluid factor attribute (FF)). The same attributes were also used to detect abrupt change in the gas hydrate structure at the points of contact with vertical gas fluid emission (gas column). It is noted that BSR completely disappears where gas hydrate crosses gas column, while intrusion of gas column into the gas hydrate layer results in appearance of complicated structures that are interpreted as mud volcanoes. The data provided in the article cannot be derived from regular interpretation of seismic sections but from AVO analysis only. 


\title{
Изучение газогидратов Берингова моря с применением скоростного и AVO анализов
}

\author{
В.В. Жигулев*(ОАО «Дальморнефтегеофизика»)
}

\section{Введение}

В связи с истощением в обозримом будущем традиционных углеводородных источников энергии возрастает актуальность совершенствования методов поиска не традиционных видов минерального топлива. В последнее время особое внимание обращено на природные газы, находящиеся в гидратном состоянии в придонной зоне акватории мирового океана. Согласно теоретическим оценкам, запасы традиционного газа оцениваются примерно в 250 триллионов тонн. В газогидратных залежах, которые содержат метан, газа как минимум в два раза больше. Если суммировать разведанные запасы нефти, газа и угля, то гидраты по перспективным ресурсам превосходят все вместе взятые объемы этих основных энергоносителей современной цивилизации.

В данной работе рассмотрены результаты изучения газогидратов с привлечением современных методов обработки сейсмических данных, включающих AVO-анализ.

\section{Методика работ}

Из всех имеющихся дистанционных способов изучения газогидратов сейсмический метод является наиболее информативным, поскольку существует прямая связь между особенностями геологического строения зоны газогидратов и сейсмоакустическими параметрами в этой среде. Индикаторами газогидратов на временных сейсмических разрезах могут быть аномальные изменения волновых сейсмических характеристик: амплитуды, частоты, фазы, скорости продольных и поперечных волн и т.д. [1-14]. Наиболее широко применяемым сейсмическим признаком присутствия в осадочном разрезе газогидрата является наличие на сейсмических разрезах горизонта, примерно повторяющего рельеф морского дна и секущего отражения от литологических границ, известного как "bottom simulation reflection" (BSR). Выделение BSR на сейсмических разрезах не вызывает затруднений в случае сечения литологических границ, но при субпараллельном залегании обнаружение этой границы требует применения дополнительных методических подходов.

Скоростной анализ с расчетом спектра скоростей для участков субпараллельного залегания границы BSR с литологическими горизонтами (рис. 1a) и
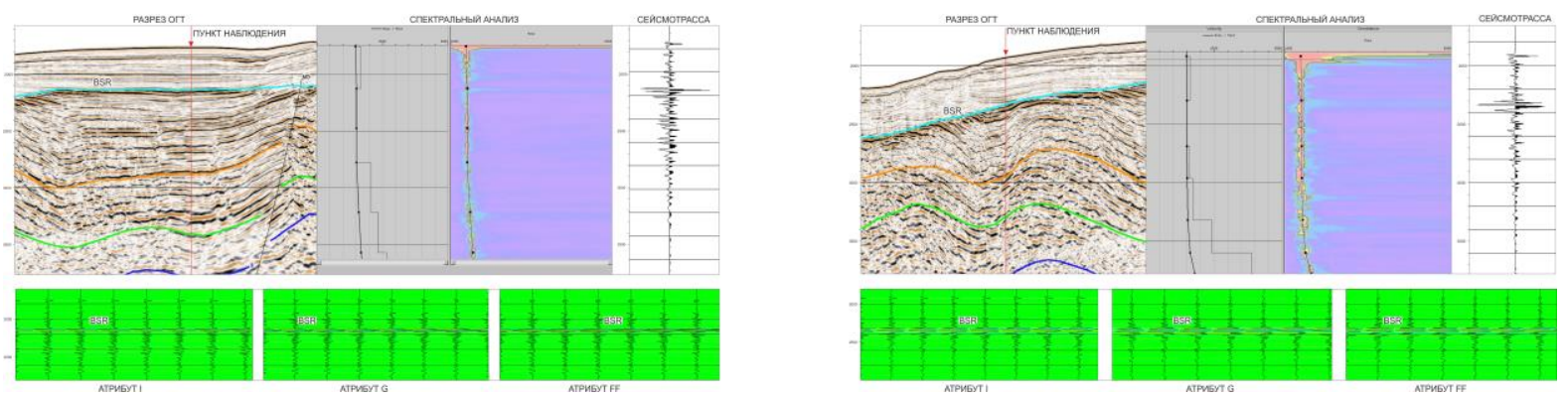

Рисунок 1. Проявление газогидрата и BSR в скоростных характеристиках и атрибутах AVO на участках параллельного (а) и секущего (б) залегания.

для участков их сечения (рис. 1б) определяет газогидратную толщу интервалом записи повышенного значения интервальной скорости относительно покрывающей водной толщи и подстилающих осадочных отложений. Резкое понижение скорости под BSR, достигающее иногда значений меньше, чем в водной толще, предположительно свидетельствует о наличии газовых скоплений. Подтверждением этого служат результаты AVO-анализа, рассчитанного по атрибутам интерцепта (I), градиента (G) и флюид-фактора (FF) (рис. 1). Доказательством наличия УВ-флюида под BSR является высокая динамика OB с фазовой инверсией по атрибуту 


\section{EAGE}

I и интенсивные отрицательные аномалии по атрибуту FF. Положительная аномалия, выделенная по атрибуту G, не характерна для УВ-содержащих песчаных коллекторов (классы $1,2,2 \mathrm{p}, 3)$ терригенного осадочного чехла региона, но вполне соответствует редкому типу газовой залежи (класс 4) в условиях, когда плотная непроницаемая масса (в нашем случае газогидрат) покрывает газосодержащие породы. Таким образом, применение скоростного и AVO-анализа позволяет достаточно надежно прослеживать границу BSR в случае, когда она не отличается от литологических границ при их конформном залегании.

Применение AVO-анализа позволило впервые исследовать предполагаемую газогидратную толщу на участках контакта с зонами вертикальной эманации газового флюида, в дальнейшем газовый столб (ГС). На рис. 2, 3, 4 представлены разрезы, иллюстрирующие характер проявления в AVO-атрибутах газогидратной толщи при различных вариантах ее взаимодействия с ГС. В случае, когда ГС насквозь пересекает газогидратную залежь с выходом флюида в водную среду (рис. 2), по всем атрибутам отчетливо наблюдается полное исчезновение BSR, внутри
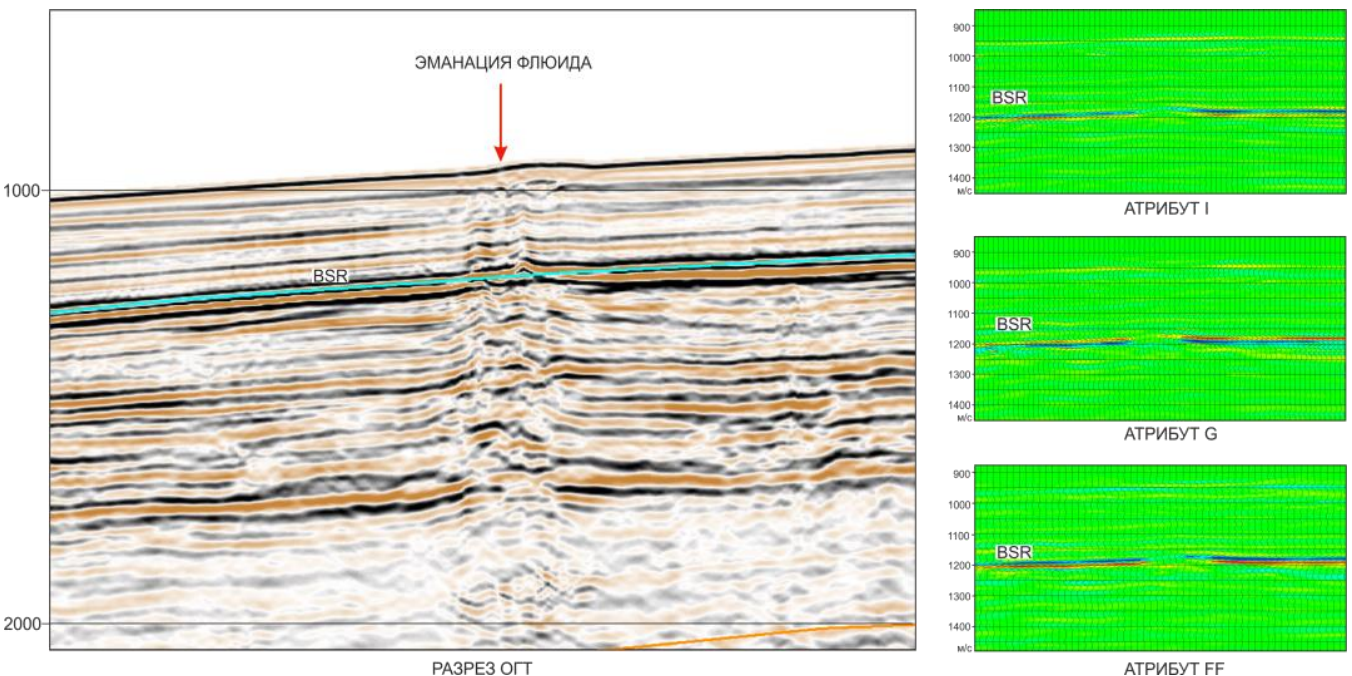

Рисунок 2. Эманация газового флюида сквозь газогидратную залежь

газогидратной толщи заметных волновых возмущений не отмечается, а на некотором удалении непосредственно под BSR прослеживаются высокоамплитудные аномалии, указывающие на присутствие УВ-флюидов. В случае, когда ГС соприкасается с подошвой газогидратной толщи, не проникая внутрь (рис. 3), граница BSR по атрибуту I не исчезает, но изменяет полярность с
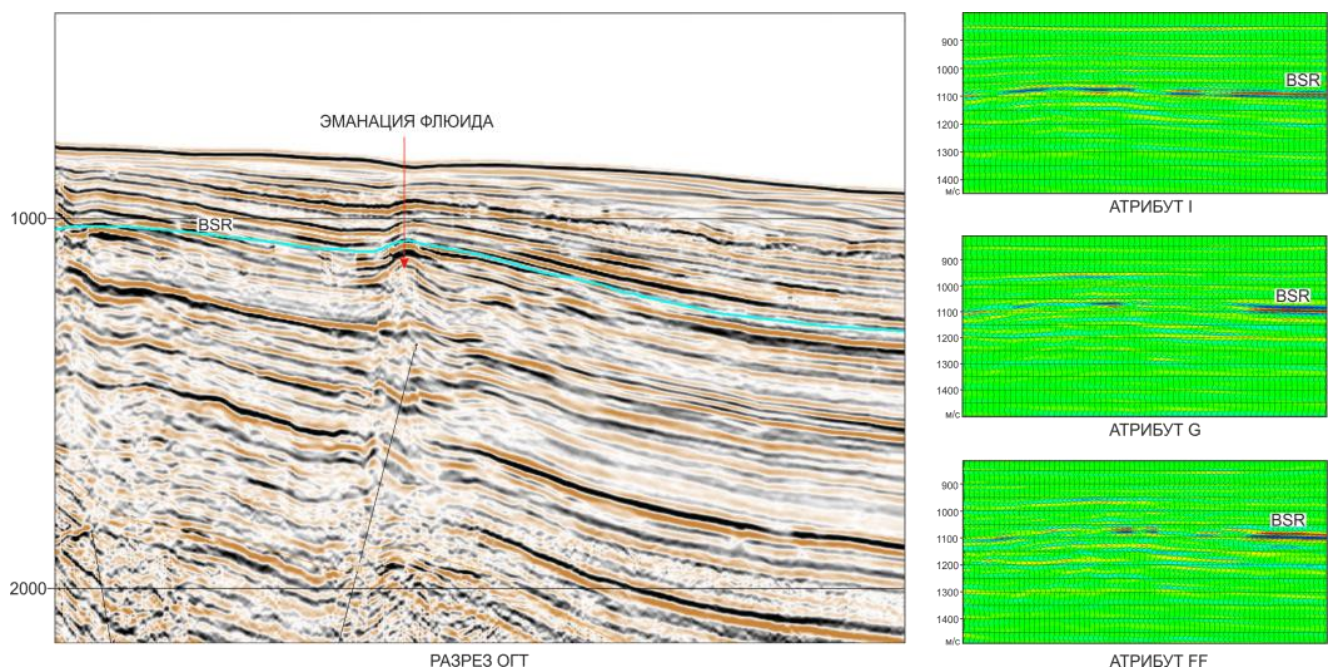

Рисунок 3. Эманация газового флюида до подошвы газогидрата 


\section{EAGE}

отрицательной на положительную, что означает переход волны от слоя с меньшей к слою с большей акустической жесткостью. По атрибутам G и FF внутри газогидрата на различных уровнях выделяются локальные включения УВ-флюидов. В случае, когда ГС внедряется внутрь газогидратной толщи, не пересекая ее насквозь (рис. 4), на разрезах атрибут I, G, FF вблизи ГС на разных уровнях образуются два горизонта BSR, которые на контакте с ГС воздымаются вверх с резким затуханием амплитуды и потерей корреляции сигналов. Образовавшееся поднятие по своей конфигурации наиболее соответствует структуре, формирующейся в результате процессов грязевого вулканизма, который наблюдается во многих районах мирового океана [14].
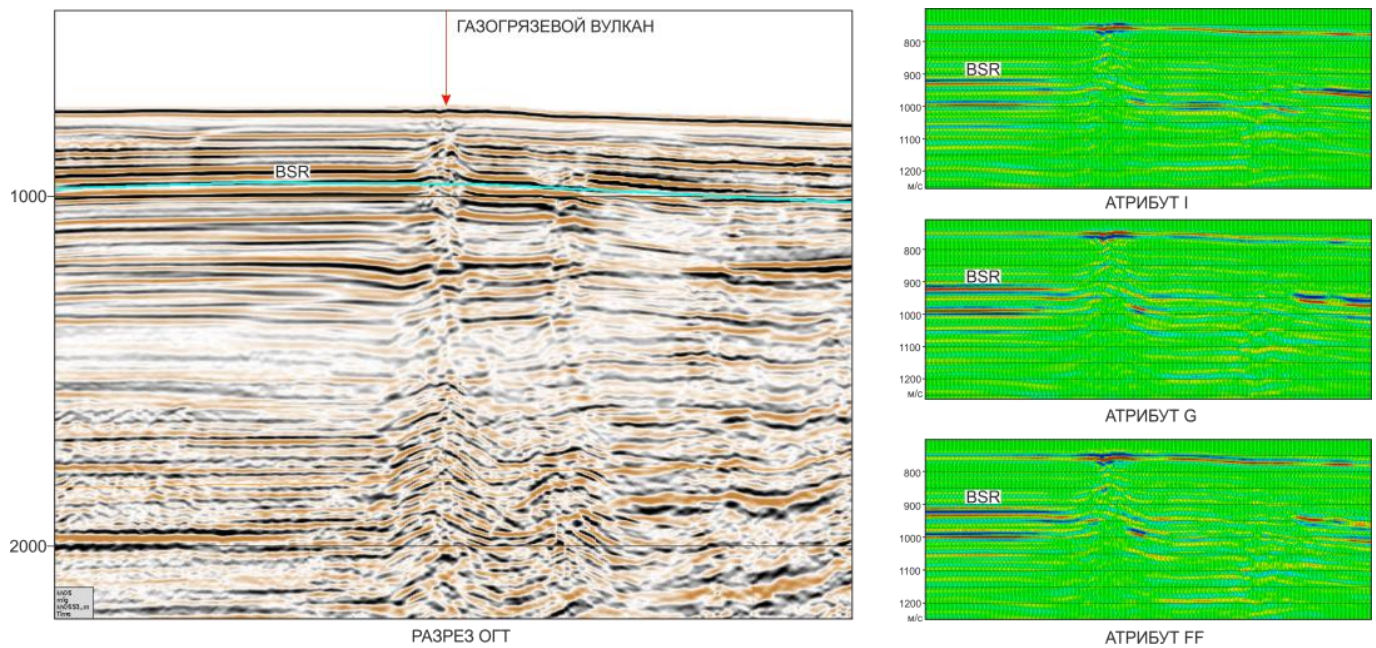

Рисунок 4. Образование газогрязевого вулкана внутри газогидратной толщии

Применение вышеизложенных критериев позволило выделить в осадочном чехле акватории западного сектора Баренцева моря зоны локализации газогидратных образований, залегающих в интервале глубин от 550 до 2380 м, мощностью от 210 до 507 м. Установлена прямая зависимость между глубиной моря и мощностью газогидратного слоя.

\section{Выводы}

Применение скоростного и AVO анализов при изучении газогидратов позволило более детально исследовать его внутреннюю структуру и, в первую очередь, подошвенный горизонт BSR, даже на тех участках сейсмического разреза, где он маскируется отражениями от литологических границ. На участках контакта газогидратной толщи с ГС установлены различные формы структурных изменений, проявляющихся в виде пронизывания насквозь ГС газогидратной толщи, образования на разных уровнях участков локализации газового флюида и формирования газогрязевых вулканических построек.

\section{Библиография}

1. Бочарова, А.А. Разработка методики картирования зон распространения газовых гидратов на основе спектрального анализа морских сейсмических данных: автореф. дисс. канд. физ.-мат. наук / А.А. Бочарова. - М.: МГУ им. М.В. Ломоносова, 2013. $25 \mathrm{c}$.

2. Гинсбург, Г.Д., Соловьев, В.А. Субмаринные газовые гидраты / Г.Д. Гинсбург, В.А. Соловьев - СПб: ВНИИ Океангеология, 1994. - 193 с.

3. Макагон, Ю.Ф. Природные газовые гидраты: распространение, модели образования, ресурсы / Ю.Ф. Макагон // Российский химический журнал. 2003. Т. 47. № 3. С. 70-79. 
4. Полетаева, Е.В. Результаты изучения региональных глубинных разломов Южного Каспия по комплексу сейсмических данных / Е.В. Полетаева // Geophysics «News in Azerbaijan». 2008. № 2. C. 25-27.

5. Хаттон, Л., Уэрдингтон, М., Мейкин, Дж. Обработка сейсмических данных / Л. Хаттон, М. Уэрдингтон, Дж. Мейкин. - М.: Мир, 1989. - 216 с.

6. Ampilov Yu.P. From seismic interpretation to modelling and Assesment of oil and Gas fields./Yu.P. Ampilov. - EUROPEAN ASSOCIATION GEOSCIENTISTS \& ENGINEERS. EAGE Publications bv, 2010, 1-274

7. Ampilov Yu.P. Baturin D.G. Latest marine geophysical technologies for comprehensive study of natural hydrocarbon reservoirs and monitoring /Yu.P. Ampilov, D.G. Baturin. - Society of Petroleum Engineers - SPE Russian Oil and Gas Exploration and Production Technical Conference and Exhibition 2012, 2056-2063

8. Diaconescu, C.C., Kieckhefer, R.M., Knapp, J.H. Geophysical Evidence for Gas Hydrates in the Deep Water of the South Caspian Basin, Azerbaijan / C.C. Diaconescu, R.M. Kieckhefer, J.H. Knapp // Marine and Petroleum Geology. 2001. № 18. P. 209-221.

9. Sain, K., Gupta, H. Gas Hydrates in India: Potential and Development / K. Sain, H. Gupta // Gondwana Research. 2012. Vol. 22. Issue 2. № 9. P. 645-657.

10. Frye, M., Shedd, W., Boswell, R. Gas Hydrate Resource Potential in the Terrebonne Basin, Northern Gulf of Mexico / M. Frye, W. Shedd, R. Boswell // Marine and Petroleum Geology. 2012. № 6. P. 150-168.

11. Thakur, N., Rajput, S. Exploration of Gas Hydrates. Geophysical Techniques / N. Thakur, S. Rajput. - Berlin-Heidelberg: Springer-Verlag, 2011.

12. Bangs, N., Hornbach, M., Berndt, C. The Mechanics of Intermittent Methane Venting at South Hydrate Ridge Inferred From 4D Seismic Surveying / N. Bangs, M. Hornbach, C. Berndt // Earth and Planetary Science Letters. 2011. Vol. 310. P. 105-112.

13. Sloan, E.D., Koh, A.C. Clathrate Hydrates of Natural Gases / E.D. Sloan, A.C. Koh. - New York: CRC Press, 2007. - 752 p.

14. Chow J.,. Lee J. S, Sun R., Liu C. S., Lundberg N. Characteristics of the bottom simulating reflectors near mud diapirs: offshore southwestern Taiwan.// Geo-Marine Letters. 2000. 20: P 3-9.

\section{References}

1. Bocharova, A.A. Development of method for mapping zones of gas hydrates spreading on the basis of spectral analysis of marine seismic data: author's abstract of Cand. Sci. (Physics and Mathematics) dissertation / A.A. Bocharova. - M: Lomonosov Moscow State University, 2013. $-25 \mathrm{p}$.

2. Ginsburg, G.D., Soloviev, V.A. Submarine gas hydrates / G.D. Ginsburg, V.A. Soloviev $\mathrm{SPb}$ : VNIIOkeangeologia, 1994. - $193 \mathrm{p}$.

3. Makogon, Yu. F. Natural gas hydrates: spreading, generation models, resources / Yu. F. Makogon // Russian Chemical Bulletin. 2003. V. 47. No. 3. P. 70-79.

4. Poletaeva, E. V. Findings of studies of deep faults in South Caspian Sea on the basis of seismic data. / E.V. Poletaeva // Geophysics "News in Azerbaijan". 2008. No. 2. P. 25-27.

5. Hatton, L., Worthington, M., Makin, J. Seismic data processing / L. Hatton, M. Worthington, J. Makin. - M.: Mir, 1989. - 216 p.

6. Ampilov Yu.P. From seismic interpretation to modelling and Assesment of oil and Gas fields./Yu.P. Ampilov. - EUROPEAN ASSOCIATION GEOSCIENTISTS \& ENGINEERS. EAGE Publications bv, 2010, 1-274

7. Ampilov Yu.P. Baturin D.G. Latest marine geophysical technologies for comprehensive study of natural hydrocarbon reservoirs and monitoring /Yu.P. Ampilov, D.G. Baturin. - Society of Petroleum Engineers - SPE Russian Oil and Gas Exploration and Production Technical Conference and Exhibition 2012, 2056-2063 
8. Diaconescu, C.C., Kieckhefer, R.M., Knapp, J.H. Geophysical Evidence for Gas Hydrates in the Deep Water of the South Caspian Basin, Azerbaijan / C.C. Diaconescu, R.M. Kieckhefer, J.H. Knapp // Marine and Petroleum Geology. 2001. № 18. P. 209-221.

9. Sain, K., Gupta, H. Gas Hydrates in India: Potential and Development / K. Sain, H. Gupta // Gondwana Research. 2012. Vol. 22. Issue 2. № 9. P. 645-657.

10. Frye, M., Shedd, W., Boswell, R. Gas Hydrate Resource Potential in the Terrebonne Basin, Northern Gulf of Mexico / M. Frye, W. Shedd, R. Boswell // Marine and Petroleum Geology. 2012. № 6. P. 150-168.

11. Thakur, N., Rajput, S. Exploration of Gas Hydrates. Geophysical Techniques / N. Thakur, S. Rajput. - Berlin-Heidelberg: Springer-Verlag, 2011.

12. Bangs, N., Hornbach, M., Berndt, C. The Mechanics of Intermittent Methane Venting at South Hydrate Ridge Inferred From 4D Seismic Surveying / N. Bangs, M. Hornbach, C. Berndt // Earth and Planetary Science Letters. 2011. Vol. 310. P. 105-112.

13. Sloan, E.D., Koh, A.C. Clathrate Hydrates of Natural Gases / E.D. Sloan, A.C. Koh. - New York: CRC Press, 2007. -752 p.

14. Chow J.,. Lee J. S, Sun R., Liu C. S., Lundberg N. Characteristics of the bottom simulating reflectors near mud diapirs: offshore southwestern Taiwan.// Geo-Marine Letters. 2000. 20: P 3-9. 